\title{
Measurement-Based Analysis of Transmit Antenna Selection for In-Cabin Distributed MIMO System
}

\author{
Yan Zhang, ${ }^{1,2}$ Zhenghui Li, $^{2,3}$ Fengyu Luan, ${ }^{1,2}$ Limin Xiao, ${ }^{2}$ Shidong Zhou, ${ }^{1}$ and Jing Wang ${ }^{2}$ \\ ${ }^{1}$ Department of Electronic Engineering, Tsinghua University, Beijing 100084, China \\ ${ }^{2}$ Tsinghua National Laboratory for Information Science and Technology, Tsinghua University, Beijing 100084, China \\ ${ }^{3}$ Department of Communication Engineering, Xiamen University, Xiamen 361005, China
}

Correspondence should be addressed to Yan Zhang, yanz@tsinghua.edu.cn

Received 31 August 2011; Accepted 10 October 2011

Academic Editor: Yuan Yao

Copyright (C) 2012 Yan Zhang et al. This is an open access article distributed under the Creative Commons Attribution License, which permits unrestricted use, distribution, and reproduction in any medium, provided the original work is properly cited.

\begin{abstract}
Aircraft seems to be the last isolated island where the wireless access is still not available. In this paper, we consider the distributed multiple-input multiple-output (D-MIMO) system application based on measurements in aircraft cabin. The channel response matrices of in-cabin D-MIMO system are collected by using a wideband channel sounder. Channel capacities with optimum transmit antenna selections (TASs) are calculated from the measured data at different receiver positions. Then the optimum capacity results are compared to those without selection in different transmit SNR. It is shown that the TAS can lead obvious capacity gain, especially in the front and back of cabin. The capacity gain represents a decreasing trend with the transmit SNR increasing. The optimal transmit antenna subset is closely related to the transmit SNR. With the SNR increasing, more transmit antennas will be chosen for higher performance. The subset of those transmit antennas near the receiver is a reasonable choice in practical application of D-MIMO system.
\end{abstract}

\section{Introduction}

With the rapid development of wireless communications, subscribers require more convenient access service at any time. Aircraft seems to be the last remaining frontier where the wireless access is still not available $[1,2]$. Passengers want to use mobile phones and laptops to meet their business and entertainment requirements in flight. To realize the in-cabin wireless access, one basic work is to investigate the propagation model in the aircraft. Some measurements have been carried out in cabin scenario to analyze the coverage and capacity of wireless systems [2-6]. MIMO (multi-input-multi-output) technology, which can improve the capacity gain and frequency efficiency, has been widely studied. However, only a little research work has been conducted for the in-cabin application of MIMO technique. In $[5,6]$, the capacity gain has been proved for the incabin centralized MIMO system. In this presented paper, we will focus on the distributed MIMO (D-MIMO) channel characteristics. Compared with traditional centralized MIMO system, D-MIMO system can provide higher energy efficiency and fairer coverage $[7,8]$. Especially in cabin scenario, the D-MIMO system could offer the passengers equal wireless service with low power consumption. To our best knowledge, there is still no measurement-based research work on the in-cabin D-MIMO channel.

In practice, access point with more antennas has to employ more radio frequency (RF) units. Then the cost of RF equipments and the complexity of the signal processing will increase too, which limit the MIMO system application. To solve this problem, antenna selection technology is proposed. In MIMO systems, antenna selection is to choose an optimum subset of antennas for communication. It can be classified as transmit $(\mathrm{Tx})$ and receive $(\mathrm{Rx})$ antenna selections. The latter one can reduce receive antenna number and signal processing complexity. Meanwhile, some receive energy will be lost, and the channel rank will not be improved. Thus, the receive antenna selection is unable to lead capacity gain of total MIMO system [9].

In this presented paper, we will emphasize the transmit antenna selection (TAS) impacts on D-MIMO system's capacity in cabin scenario. For D-MIMO channel, because the 
transmit antennas are placed with distances, different links suffer diverse shades. Then the TAS can be useful with utilization of the macrodiversity gain. In order to validate the TAS's effect based on measured results, measurement campaigns are carried out in cabin to collect MIMO channel-impulseresponse (CIR) matrices. Based on the collected data, the capacities under different antenna selection schemes with fixed total transmit power are analyzed and compared. The results show that, besides the reduction of the complexity and cost, the TAS will lead capacity gain in cabin scenario. The relationship between the signal-to-noise ratio (SNR) and antenna selection scheme is also discussed. Then we present a simple near-optimum selecting way for practical application.

The rest of this paper is organized as follows. In Section 2, the channel sounder and measurement setup are introduced. The D-MIMO channel capacity with TAS is characterized in Section 3. In Section 4, the measurement results are shown to evaluate the TAS performance. Finally, our conclusions are presented in Section 5.

\section{Experimental Setup}

2.1. THU Channel Sounder. The Tsinghua University (THU) MIMO channel sounder [10] was used to collect raw measured data. It worked at the center frequency of $3.52 \mathrm{GHz}$ with $40 \mathrm{MHz}$ bandwidth, supporting both centralized and distributed MIMO channel measurements. During the incabin measurements, the transmitter employed a signal generator to periodically output a linear frequency modulated (LFM) sequence. A microwave switch was used to connect the signal generator with seven transmit antenna ports. Tx antennas were connected to the switch through cables and were distributed in the cabin.

At the receiver side, seven antennas constituted a uniform linear array (ULA) with half-wavelength interelement spacing. The received signal was input to one RF tunnel via another 7-way switch. Then a 7-input 7-output system was realized by adopting this fast time-division-multiplexed switching (TDMS) scheme. The microwave switches were controlled to scan all possible antenna combinations by a synchronization unit.

The major configurations of the THU channel sounder were shown in Table 1 . The test signal length $t_{p}$ was $12.8 \mu \mathrm{s}$. A guard interval $t_{p}$ was also inserted between adjacent transmission to protect the test signal from the delay spread infection. Then one total snapshot interval was $7 \times 7 \times$ $2 t_{p}=1254.4 \mu \mathrm{s}$. The real-time measured data was stored in a server. To obtain the channel parameters of interest, the data processing was finished off line.

2.2. Measurement Environment. Our measurement campaigns are carried out in an MD-82 aircraft. The MD-82 [11] is a short-haul aircraft with 149 seats arranged in 33 rows. The first three rows are the business class and the other rows are the economy class. The dimensions of the cabin are $30.5 \mathrm{~m}$ length, $3.34 \mathrm{~m}$ width, and $2.05 \mathrm{~m}$ height, respectively. The aisle width is $0.5 \mathrm{~m}$, and the distance between rows is $0.7 \mathrm{~m}$. The seat height is $1.16 \mathrm{~m}$ above the floor.
TABLE 1: The configurations of THU MIMO channel sounder.

\begin{tabular}{ll}
\hline Parameter & Range \\
\hline Carrier frequency & $3.52 \mathrm{GHz}$ \\
Bandwidth & $40 \mathrm{MHz}$ \\
Tx signal length & $12.8 \mu \mathrm{s}$ \\
Snapshot interval & $1.2544 \mathrm{~ms}$ \\
Antenna configuration & $7 \times 7$ \\
Antenna type & Omnidirectional with 4 dBi gain \\
Polarization & Vertical \\
\hline
\end{tabular}

As illustrated in Figure 1, seven transmit antennas (Tx1Tx7) were fixed at different positions. The Tx antenna height was $1.68 \mathrm{~m}$ above the floor, and the distance between adjacent Tx antennas was $2.9 \mathrm{~m}$.

The cross-section of the measurement environment was shown in Figure 2. The receiver with centralized antenna array was placed on a dining car. The receiver height was $1.37 \mathrm{~m}$. There are seven $\mathrm{Rx}$ antennas $(\mathrm{Rx} 1-\mathrm{Rx} 7)$ at the receiver, but in the following analysis only three of them were used. Other elements were used as dummy elements, and their responses were discarded.

During the measurements, the receive array's position was changed along the aisle from the 4 th row to the 27 th row of the economic class, as the pentagrams shown in Figure 1. At each position, receiver was moved in an $8 \lambda$ interval back and forth, in order to collect channel data with independent small-scale fading.

\section{D-MIMO Capacity with Transmit Antenna Selection}

3.1. Distributed MIMO Channel Model. In D-MIMO system, each base station has $N$ distributed antenna ports, each port with $V$ microdiversity antennas. The mobile station's antenna number is $M$, and then this D-MIMO system can be noted by $(N, V, M)[12]$.

This $(N, V, M)$ D-MIMO channel can be described as

$$
\mathbf{H}=\left[\mathbf{H}_{1}\left(d_{1}\right), \mathbf{H}_{2}\left(d_{2}\right), \ldots, \mathbf{H}_{N}\left(d_{N}\right)\right]_{M \times N V},
$$

where $d_{i}(i=1, \ldots, N)$ is the distance between the $i$ th antenna port to the mobile station. $\mathbf{H}_{i}\left(d_{i}\right)$ is the $M \times V$ CIR matrix of $i$ th antenna port. If $N=1$, it becomes a traditional centralized system, and if $V=1$ a star-shaped D-MIMO. As introduced in Section 2, our channel sounder is a $(7,1,7)$ star-shaped D-MIMO system.

3.2. D-MIMO Capacity with Transmit Antenna Selection. Consider a channel is unknown at the transmitter. The capacity of a D-MIMO channel with equally allocated transmit power can be calculated as [9]

$$
C=\log _{2} \operatorname{det}\left(\mathbf{I}_{M}+\frac{P_{t}}{N \sigma^{2}} \mathbf{H H}^{H}\right) .
$$

Here, $\mathbf{H}$ is the $M \times N$ CIR matrix, whose elements include the pathloss, shade fading, and small-scale fading effects. 


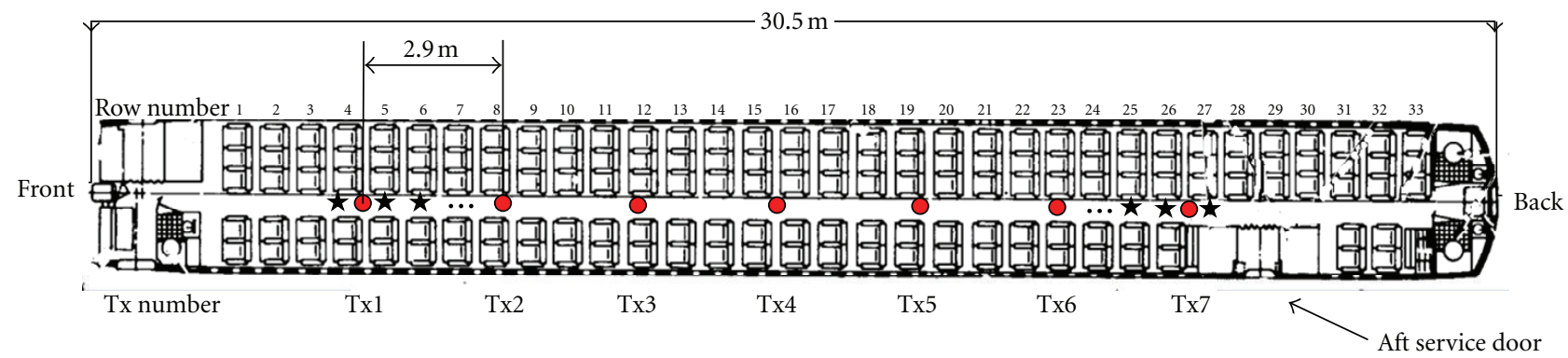

Tx antenna position

$\star$ Rx array position

FIGURE 1: Interior arrangement of the measurement environment.

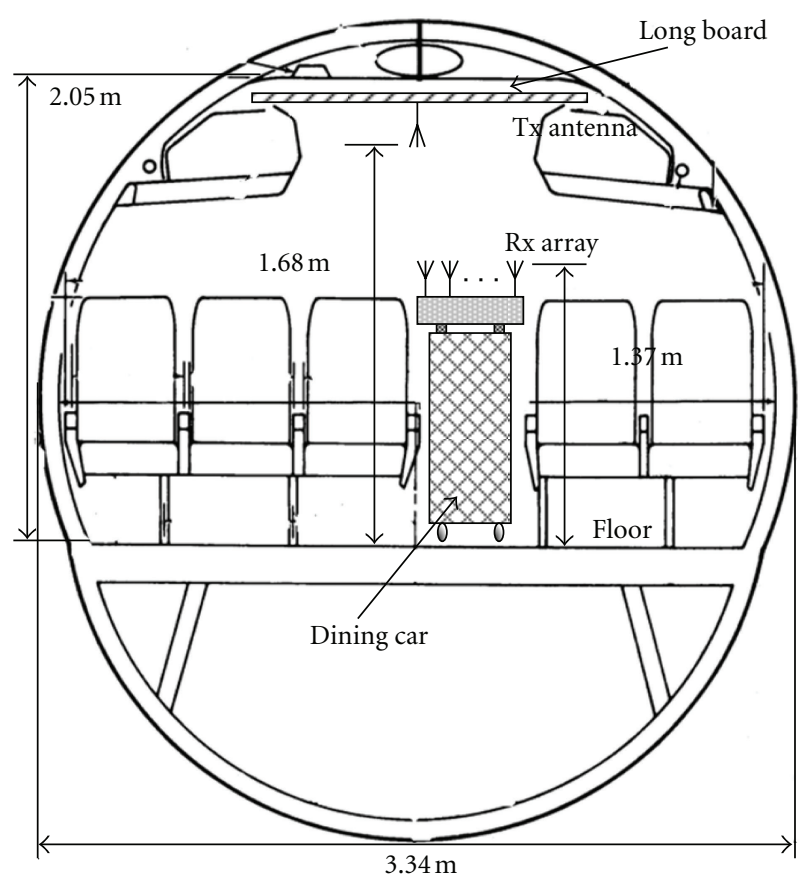

Figure 2: Cross-section of the measurement environment.

Then it is not a normalized matrix. $N$ and $M$ are the transmit and receive antenna numbers, respectively. $\mathbf{I}_{M}$ is an $M \times M$ identity matrix. $P_{t}$ is the total transmit power and $\sigma^{2}$ is the noise power. Then $\rho_{t}=P_{t} / \sigma^{2}$ is the transmit SNR. Superscript $(\cdot)^{H}$ denotes the Hermitian transpose.

Two metrics including ergodic capacity and outage capacity are usually used to evaluate MIMO system's performance. The ergodic capacity is corresponding to the average capacity of random channel and will be considered in the following analysis. The ergodic capacity can be computed by

$$
C_{\text {ergodic }}=E_{\mathbf{H}}\left\{\log _{2} \operatorname{det}\left(\mathbf{I}_{M}+\frac{P_{t}}{N \sigma^{2}} \mathbf{H H}^{H}\right)\right\} .
$$

$E_{\mathrm{H}}(\cdot)$ is the average operator. It means that independent channel realizations are needed to obtain the ergodic capacity. For each Rx position's data processing, we consider both the spatial realizations in $8 \lambda$ and the frequency realizations in $40 \mathrm{MHz}$ bandwidth.

The objective of TAS is to choose an optimum antenna subset including $L$ antennas to maximize capacity given by

$$
C_{\text {sel }}=\max _{\widetilde{\mathbf{H}} \in \mathbf{H}}\{C\}=\log _{2} \operatorname{det}\left(\mathbf{I}_{M}+\frac{P_{t}}{L \sigma^{2}} \tilde{\mathbf{H}} \tilde{\mathbf{H}}^{H}\right),
$$

where $\widetilde{\mathbf{H}}$ is an $M \times L$ subblock matrix of $\mathbf{H}$.

The capacity gain with antenna selection can be defined as

$$
G=\frac{C_{\text {opt }}-C_{\text {no_sel }}}{C_{\text {no_sel }}} .
$$

Here, $C_{\text {opt }}$ is the ergodic capacity with optimum selection subset. $C_{\text {no_sel }}$ is the capacity without antenna selection; that is, all transmit antennas are employed to send equal power.

\section{Measurement Results}

4.1. Antenna Selection Effect on Channel Capacity. As mentioned above, the channel sounder is a star-shaped D-MIMO system with seven distributed transmit antennas. The receiver also employs seven $\mathrm{Rx}$ antennas as a centralized array. In future wireless systems, limited to the size and cost requirements, three or fewer antennas are practicable for most mobile terminals (e.g., mobile phones). So we only select three receive antennas $(\mathrm{Rx} 3, \mathrm{Rx} 4$, and $\mathrm{Rx} 5)$ for following analysis. These three antennas are located at the center of the array, and one-wavelength array size is approximately a mobile phone's length. Then we will focus on a $(3,7,1)$ D-MIMO system's performance with TAS.

According to (3), firstly the ergodic capacities without antenna selection are extracted from the measured data at 24 different positions. Then the max capacity with TAS is computed by searching all possible antenna selection schemes. To keep the comparison justice, the total transmit power of all $\mathrm{Tx}$ antennas is set to be equal for a fair comparison. If less antennas are used, each antenna will radiate more power. As illustrated in Figure 3, TAS can lead obvious capacity gain. Here the transmit SNR is $87 \mathrm{~dB}$, which is corresponding to about $20 \mathrm{~dB}$ average receive SNR. For all receiver positions, the gains are between $3 \%$ and $14 \%$. 


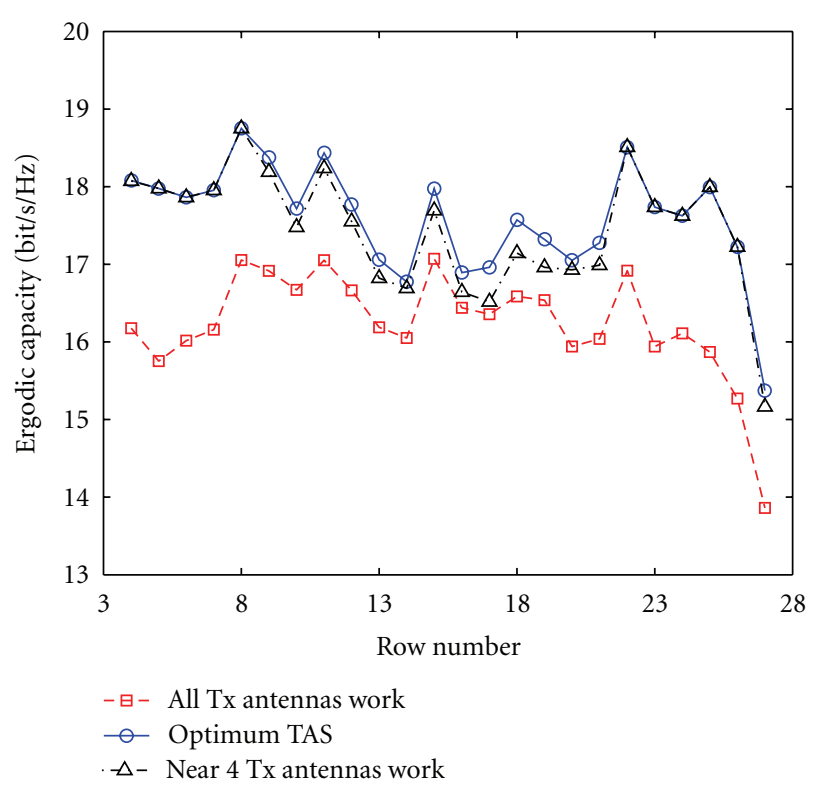

Figure 3: Ergodic capacities with different TAS schemes at $24 \mathrm{Rx}$ positions.

This capacity gain is related to the $\mathrm{Rx}$ locations. In this cabin scenario, the distributed Tx antennas are arranged in a line. When all transmit antennas work together with equal power, some of them are far from the receiver and energy from these antennas are small. Thus, the receive SNR is lower than that with TAS. For example, at the front and back of the cabin, antennas at the other side are far from the receiver, so the capacity with TAS can be $10 \%$ more than that without selection. In the middle of cabin, because the distances between receiver and different $\mathrm{Tx}$ antennas are similar, the capacity gain brought by TAS is relatively smaller.

Moreover, in the front or back of the cabin, the macro diversity is smaller than that in the middle. In our previous work [13], we have proved that when $\mathrm{Rx}$ is placed in the cabin back (e.g., the 25th row), the signals sent from Tx1 and Tx2 would undergo similar reflection and scattering, which leads to strong spatial correlation between Tx1 and Tx2. Similar thing occurs when receiver is located in the front of cabin. Then the microdiversity effects led by distributed Tx antennas are not large in the front or back of cabin. Comparatively, in the middle the Tx correlations are smaller. Selecting more Tx antennas will increase the channel rank obviously and make the eigenvalues more uniform.

Equation (2) can be rewritten as

$$
C=\sum_{j=1}^{\operatorname{rank}(\mathbf{H})} \log _{2}\left(1+\frac{P_{t}}{N \sigma^{2}} \lambda_{j}\right),
$$

where $\operatorname{rank}(\mathbf{H})$ is the rank of the CIR matrix $\mathbf{H} . \lambda_{j}$ is the $j$ th eigenvalue. According to (6), the improvement of channel rank and min eigenvalue can partly counteract the receive energy loss. Thus, the gap between the two curves is not large when $\mathrm{Rx}$ is in the middle.

The capacities measured at the 26th and 27th rows are lower than others. It may be led by the energy leakage because

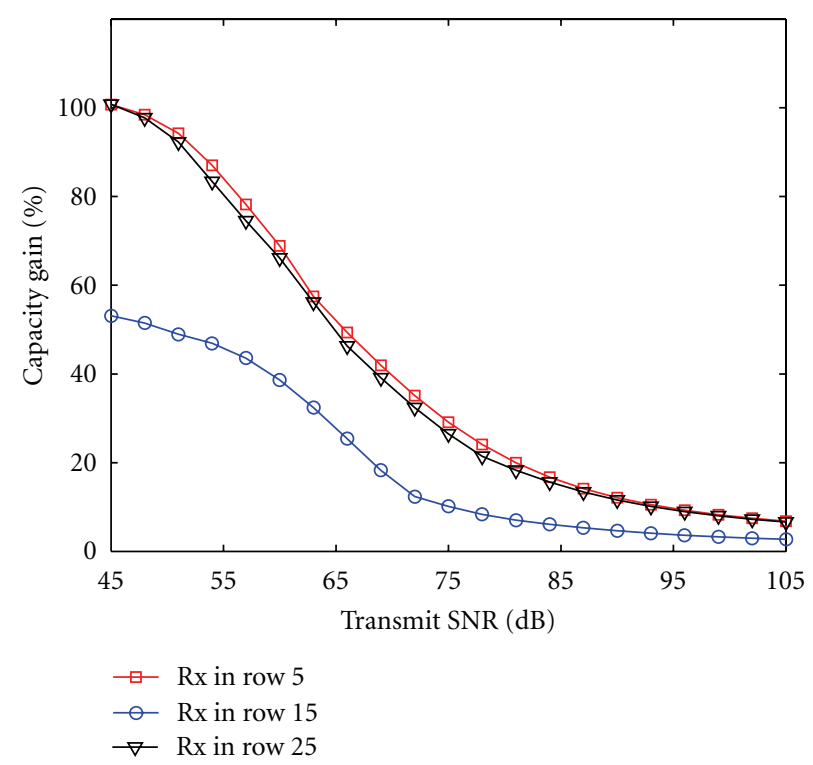

FIGURE 4: TAS capacity gains with different transmit SNRs using $\mathrm{Rx} 3, \mathrm{Rx} 4$, and $\mathrm{Rx} 5$.

the Aft service door was always open during measurement campaigns.

In Figure 4, the capacity gains with TAS at different SNRs are illustrated. It can be seen that capacity gain falls with the increase of transmit SNR. Similarly, selecting more transmit antennas will improve the CIR matrix rank. According to (6), with high transmit SNR, larger CIR matrix rank and more uniform eigenvalues can increase the channel capacity without TAS. For practical communication systems, the receive SNR is usually from $-5 \mathrm{~dB}$ to $20 \mathrm{~dB}$, approximately corresponding to the transmit SNR from $60 \mathrm{~dB}$ to $85 \mathrm{~dB}$. In this range, the capacity gain is visible. Also, it can be seen that the capacity gain at the middle is smaller than those at the edges of the cabin.

4.2. Optimum Transmit Antenna Selection Scheme. With different transmit SNRs, the proportions of the optimal antenna number at all 24 positions are shown in Figure 5. It can be seen that with very low SNR, for example, lower than $50 \mathrm{~dB}$, at most positions only one antenna is needed to reach the largest capacity. With the increase of transmit SNR, the smallest eigenvalue of CIR matrix becomes bigger, and then more antennas can provide better performance by leading larger multiplexing effect. In this situation, more antennas can do that. Then the capacity will become larger. However, even with very high SNR, the probability of selecting six or more antennas is very small. In the application of the D-MIMO system, it means that all transmit antennas are not necessary for one passenger's service. Considering the practical receive $\mathrm{SNR}$, the number of the transmitter $\mathrm{RF}$ devices can be reduced. In most cases, receiver with three antennas only needs from 2 to 4 transmit antennas.

Then another question is which antenna subset is the optimum one. For seven transmit antennas, there are totally 127 selection schemes. Searching all combinations will 


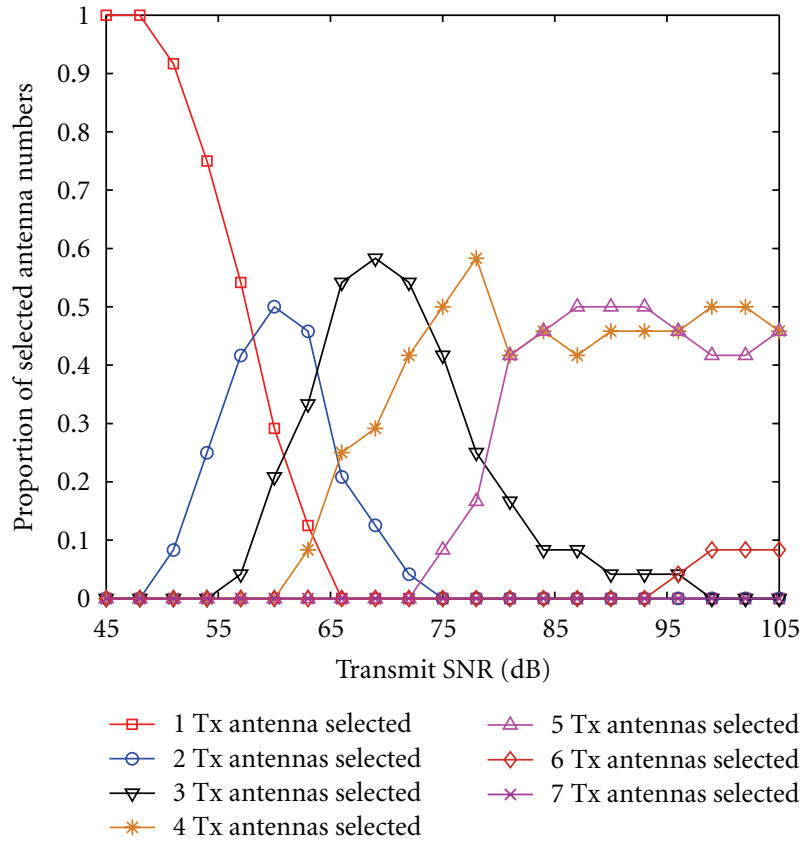

FIgUre 5: The antenna number of optimal TAS subset.

lead high processing complexity. According to the former analysis, we notice that the access distance is quite important for D-MIMO system. Then one near-optimum scheme is to select transmit antennas near the receiver. As an example, in Figure 3 we give the results when using about four transmit antennas. The black dot-dash with triangle marker is corresponding to the ergodic capacity at this case. It can be seen that this TAS scheme's performance is quite close to the optimum one. Then in practical application, we can just select the nearest 2 to 4 antennas to serve the passengers in different rows.

4.3. Results with Larger Receive Array. In the analysis above, we choose Rx3, Rx4, and Rx5 to form a small-size (onewavelength) receive array. Small array means that there exist high correlations among receive antennas in the long and narrow cabin. The CIR matrix has low rank, and the eigenvalues distribute nonuniformly, especially at the ends of the cabin. In (6), it means that the SNR is the deterministic factor to the channel.

If we consider a larger receive array, including $\mathrm{Rx} 1, \mathrm{Rx} 4$, and Rx7. This array is 3-wavelength long, which is corresponding to a laptop's size. Then Rx correlations are smaller. Choosing more transmit antennas will make the eigenvalues distribute more uniform, which can partly eliminate the loss of energy. Thus in this situation, the capacity gain with TAS will be lower. The measured results also prove that, as shown in Figure 6. Compared with Figure 4, the capacity gain is not larger than $40 \%$. The differences between the middle and edges are not so large as that in Figure 4.

\section{Conclusion}

The TAS effects on channel capacity of in-cabin D-MIMO system were analyzed by using measured data. The optimum

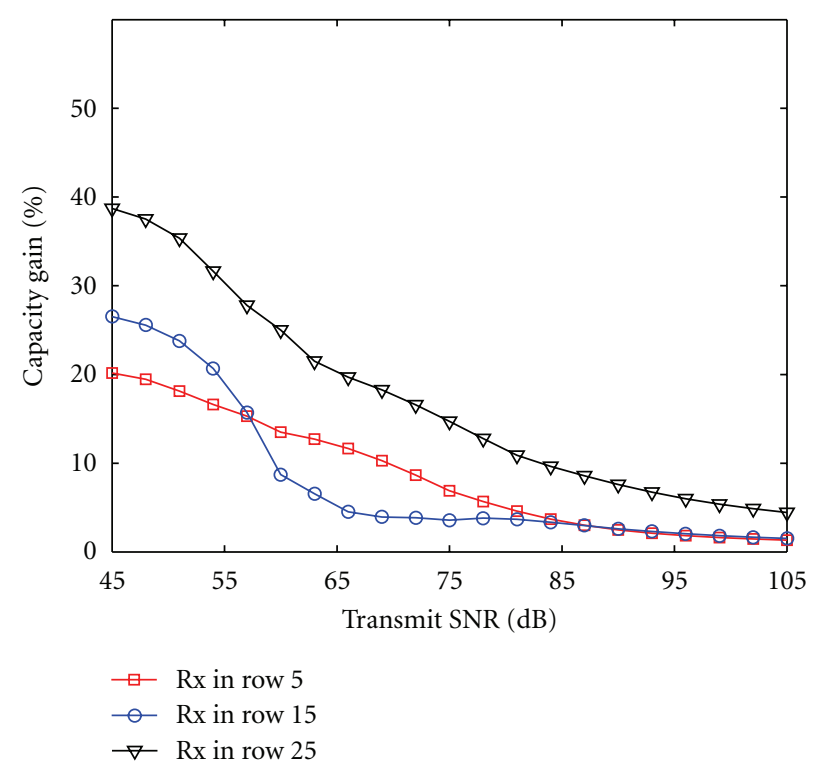

FIGURE 6: TAS capacity gain with different transmit SNR using Rx1, $\mathrm{Rx} 4$, and $\mathrm{Rx} 7$.

TAS subsets at different Rx positions were found out by searching all possible schemes. Then the ergodic capacity with optimum TAS was compared with that without selection. The results showed that the TAS could lead visible capacity gain, especially in the front and back of the cabin. With the increase of the transmit SNR, the capacity gain decreased. The selected antenna number in the optimum subset also depended on the transmit SNR. With low SNR only few antennas were needed to provide better performance, while in high SNR condition more antennas would lead larger capacity. And in most cases, the optimum selected number was smaller than 5 . One practical selection scheme was to choose the Tx antennas near the receiver. For receiver with larger array size, the effects of TAS would become smaller. These results provided practical references to the future distributed MIMO system applications in cabin.

\section{Acknowledgments}

This work was partially supported by National Basic Research Program of China (2012CB316002), Tsinghua University Initiative Scientific Research Program (20111081025), China's Major Project (2009ZX03007-003-02), China's 863 Project (2009AA011501), PCSIRT, Tsinghua-Qualcomm Joint Research Program, and China Postdoctoral Science Foundation.

\section{References}

[1] A. Jahn, M. Holzbock, J. Müller et al., "Evolution of aeronautical communications for personal and multimedia services," IEEE Communications Magazine, vol. 41, no. 7, pp. 36-43, 2003.

[2] N. Moraitis, P. Constantinou, F. P. Fontan, and P. Valtr, "Propagation measurements and comparison with EM techniques 
for in-cabin wireless networks," Eurasip Journal on Wireless Communications and Networking, vol. 2009, Article ID 784905, 13 pages, 2009.

[3] N. R. Díaz and J. E. J. Esquitino, "Wideband channel characterization for wireless communications inside a short haul aircraft," in Proceedings of the IEEE 59th Vehicular Technology Conference (VTC '04-Spring), pp. 223-228, Milan, Italy, May 2004.

[4] A. Kaouris, M. Zaras, M. Revithi, N. Moraitis, and P. Constantinou, "Propagation measurements inside a B737 aircraft for in-cabin wireless networks," in Proceedings of the IEEE 67th Vehicular Technology Conference-Spring, VTC, pp. 2932-2936, Singapore, May 2008.

[5] J. R. Nagel, A. M. Richards, S. Ananthanarayanan, and C. M. Furse, "Measured multi-user MIMO capacity in aircraft," IEEE Antennas and Propagation Magazine, vol. 52, no. 4, Article ID 5638282, pp. 179-184, 2010.

[6] R. Bhagavatula, R. W. Heath Jr, and S. Vishwanath, "Optimizing MIMO antenna placement and array configurations for multimedia delivery in aircraft," in Proceedings of the IEEE 65th Vehicular Technology Conference (VTC2007-Spring), pp. 425429, Dublin, Ireland, April 2007.

[7] M. Alatossava, A. Taparugssanagorn, V. Holappa, and J. Ylitalo, "Measurement based capacity of distributed MIMO antenna system in Urban microcellular environment at 5.25 GHz," in Proceedings of the IEEE Vehicular Technology Conference (VTC Spring 2008), pp. 430-434, May 2008.

[8] X. Hu, Y. Zhang, Y. Jia, S. Zhou, and L. Xiao, "Power coverage and fading characteristics of indoor distributed antenna systems," in Proceedings of the 4th International Conference on Communications and Networking in China (CHINACOM '09), pp. 1-4, Xian, China, August 2009.

[9] S. Sanayei and A. Nosratinia, "Capacity of MIMO channels with antenna selection," IEEE Transactions on Information Theory, vol. 53, no. 11, pp. 4356-4362, 2007.

[10] Y. Zhang, X. Hu, Y. Jia, S. Zhou, X. Chen, and J. Wang, "A novel coupling-based model for wideband MIMO channel," in Proceedings of the Global Telecommunications Conference (GLOBECOM '09), pp. 1-6, Honolulu, Hawaii, USA, 2009.

[11] http://www.boeing.com/commercial/airports/m80.htm.

[12] J.-K. Lain, "Joint transmit/receive antenna selection for MIMO systems: a real-valued genetic approach," IEEE Communications Letters, vol. 15, no. 1, pp. 58-60, 2011.

[13] Z. H. Li, F. Y. Luan, Y. Zhang, L. M. Xiao, L. F. Huang, and S. D. Zhou, "Capacity and spatial correlation measurements for wideband distributed MIMO channel in aircraft cabin environment," in Proceedings of the IEEE Wireless Communications and Networking Conference (WCNC'12), Paris, France, 2012. 

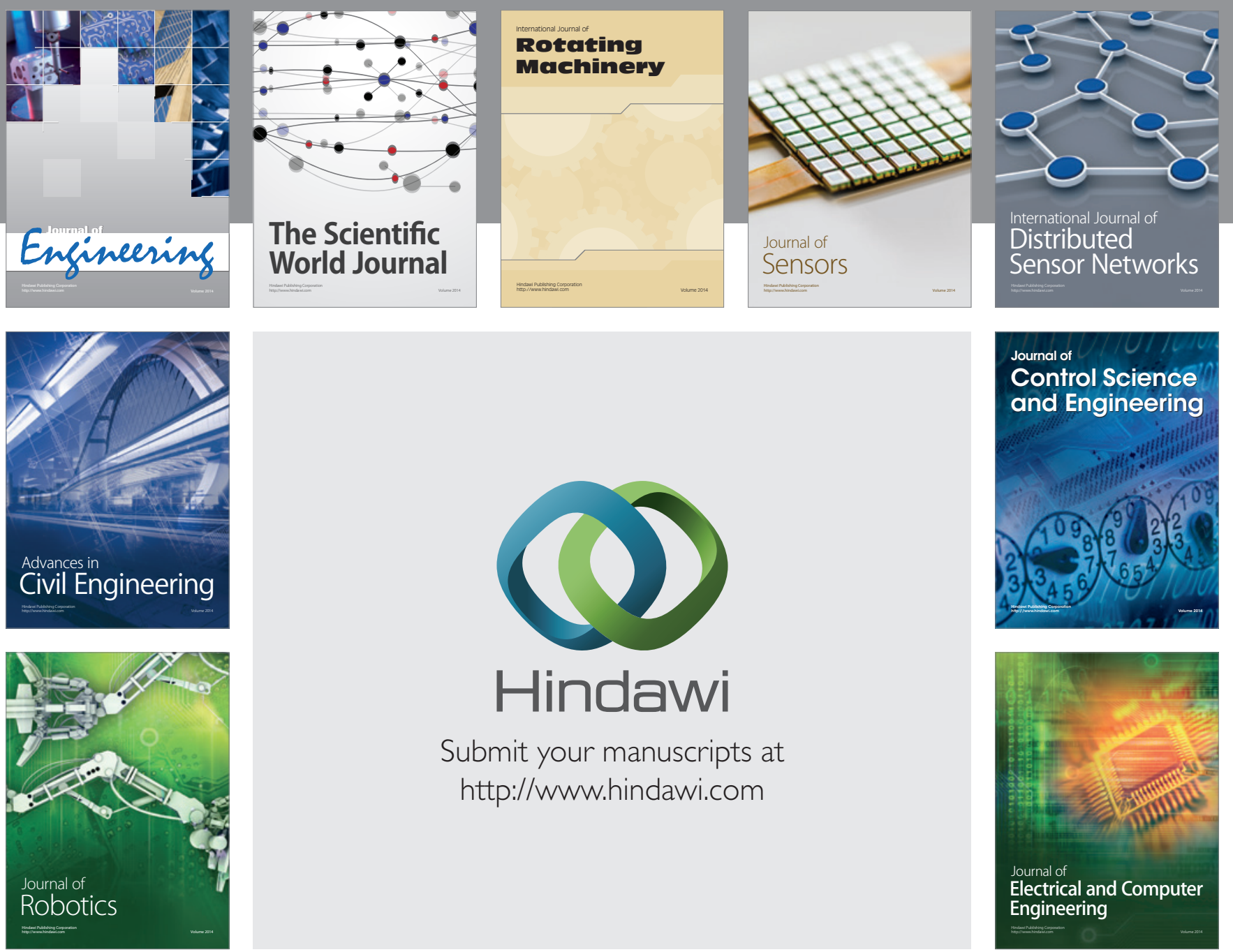

Submit your manuscripts at

http://www.hindawi.com
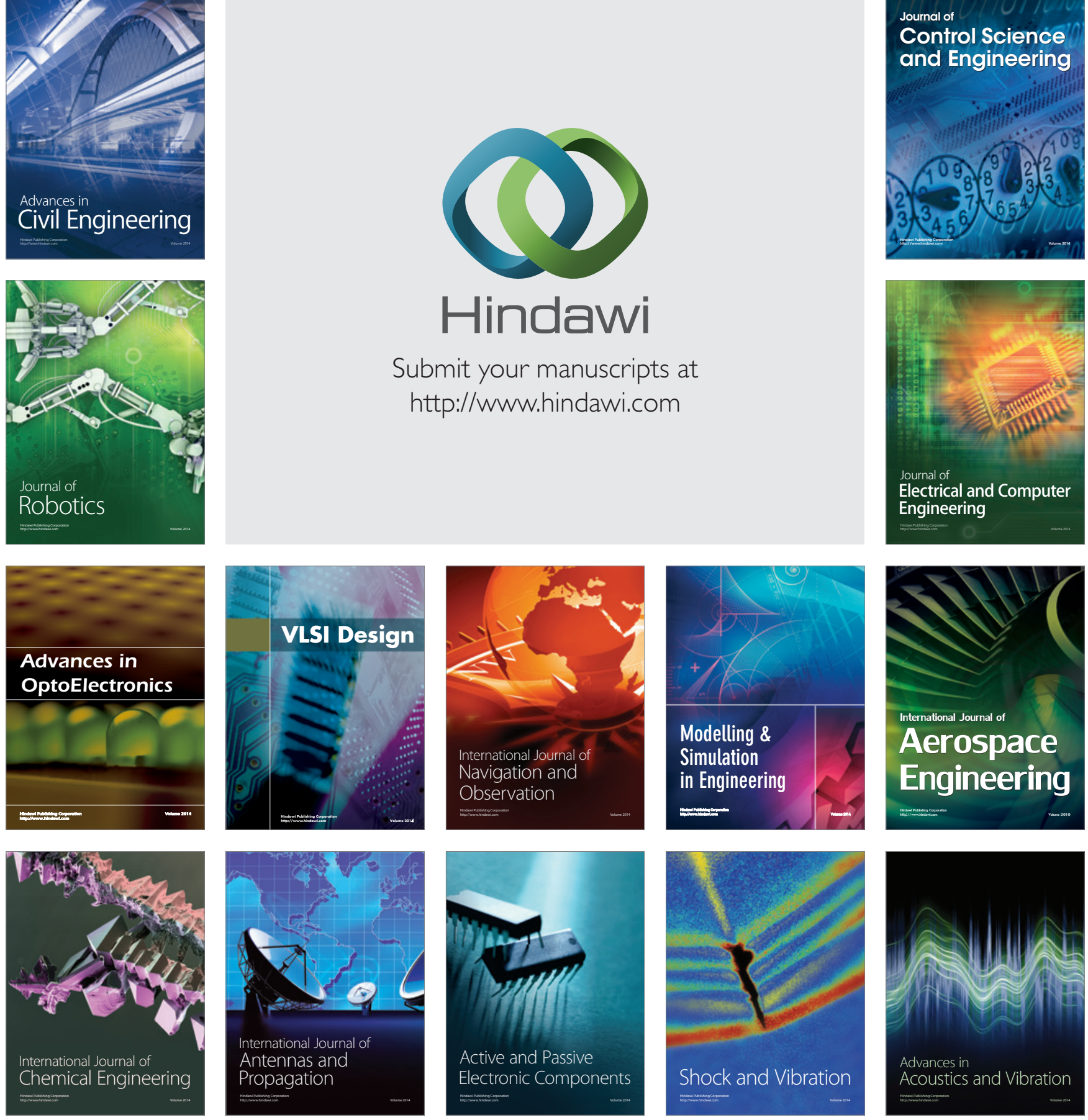